\title{
The Sri Lanka National Blindness, Visual Impairment and Disability Survey: rationale, objectives and detailed methodology
}

\author{
G V S Murthy ${ }^{1,2}$, C Gilbert ${ }^{2}$, E Schmidt ${ }^{3}$, P G Mahipala ${ }^{4}, K_{\text {M K Gamage }}^{5}$, C Banagala ${ }^{5}$, A P Abeydeera 6 , \\ A Jeza ${ }^{6}$ on behalf of the Sri Lanka National Blindness, Visual Impairment and Disability Steering \\ Committee and Survey Team ${ }^{7}$
}

\begin{abstract}
Introduction Population-based data on visual impairment are required to estimate the need for services, evaluate service delivery and identify priorities that need to be addressed, as recommended in the World Health Organisation (WHO) Global Action Plan for Universal Eye Health. In the absence of national data for Sri Lanka, a nationwide survey was initiated.
\end{abstract}

Objectives Determine the prevalence, distribution, magnitude and causes of blindness, visual impairment amongst adults aged $\geq 40$ years in Sri Lanka; and to determine risk factors for blindness and visual impairment, and disability from the major causes.

Methods The survey recruited a nationally representative sample of adults aged $\geq 40$ years using proportionateto-population size methods and $\geq 18$ years to estimate the prevalence of disability. All nine provinces and 25 districts of the country were included. 100 participants aged $\geq 40$ years were enumerated in each of the randomly selected 68 clusters, giving a sample of 6800 persons. An examination site was set up in each cluster with space for interviews, anthropometry and clinical examination.

Results Fieldwork took place over a year from 2013 to 2014. Written informed consent was obtained before the examination. Overall response rate was $86.1 \%$. Companion articles in this issue highlight the prevalence and causes of blindness, visual impairment and disability and the role of socio-demographic factors in the prevalence estimates.

Conclusions The findings from the survey can be extrapolated to the entire country as the sample was representative of the demographic characteristics of Sri Lanka. Data provides sufficient evidence for program planners to identify priority regions for specific interventions to reduce the prevalence of blindness, visual impairment and disability.

Ceylon Medical Journal 2018; 63 (S2): s3-s9

DOI: http://doi.org/10.4038/cmj.v63i5.8735

\section{Introduction}

Sri Lanka is unique in South and South-East Asia. It's Human Development Index (HDI) at 0.757 [1], is the highest of all countries in South/South-East Asia [2]. Sri Lanka is home to 21.27 million people, with $51.6 \%$ being female (2013) [3]. Life expectancy in Sri Lanka is the highest in South Asia and the infant mortality rate of 9.9 per 1000 live births points to a health status like many high-income countries [4]. Sri Lanka has one of the fastest ageing populations in the region [3]. The country is divided into nine provinces and 25 districts (Figure 1).

The government provides free health care, including eye care. The VISION2020 (a global initiative to eliminate the major causes of avoidable blindness by the year 2020) country prevention of blindness programme was launched by the Ministry of Health and covers five main conditions, namely cataract, glaucoma, blindness in children, diabetic retinopathy, refractive errors and low vision [5].

The World Health Organization (WHO) estimates 285 million people visually impaired, including 39 million blind, globally [6]. The principal causes of visual impairment are uncorrected refractive errors (43\%) and cataract (33\%); and the commonest cause of blindness is cataract $(51 \%)[6]$.

${ }^{1}$ Indian Institute of Public Health, Hyderabad, India, ${ }^{2}$ Department of Clinical Research, London School of Hygiene and Tropical Medicine, ${ }^{3}$ Sightsavers UK, Haywards Health, West Sussex, UK, ${ }^{4}$ Director General Health Services, Ministry of Health, Sri Lanka, ${ }^{5}$ Sri Lanka College of Ophthalmologists, Colombo, Sri Lanka, ${ }^{6}$ Vision2020 Coordination Committee, Ministry of Health, Govt. of Sri Lanka, ${ }^{7}$ In addition to the above, other members of the Sri Lanka National Blindness, Visual Impairment and Disability Steering Committee and Survey Team: Kapila Edussuriya, Saman Senanayake, Sunil Fernando, Lakmini Dissanayake, Nirmi Vitharana, Nimal Edirisinghe, Sunil Settinayake, RP Kumara, SAHK Wimalarathne, Attapathu AH, Priyangani MD, Bandara KRTC, Chamin Rathnayake, YG Upali Jayarathne, Souvik Bandhopadhyaya, Hira Ballabh Pant, Mahesh Dorairaj, Sandeep Bhuttan.

Correspondence: GVSM, e-mail: <Gvs.Murthy@lshtm.ac.uk>. Received 19 February 2018 and revised version accepted 23 June 2018.

(i) This is an open-access article distributed under the terms of the Creative Commons Attribution License, which permits unrestricted use, distribution, and reproduction in any medium, provided the original author and source are credited. 


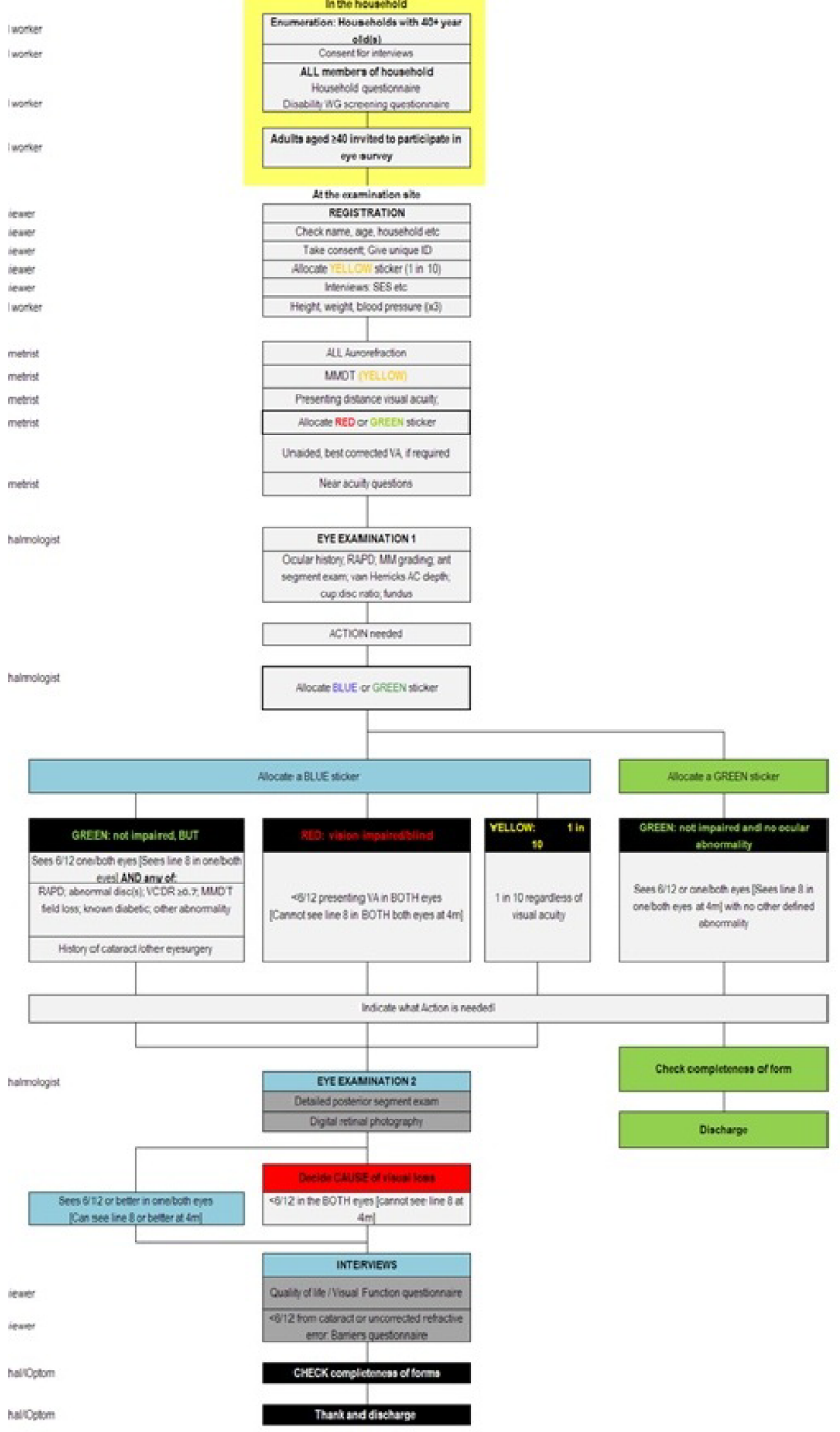

Figure 1. Flowchart describing the protocol adopted in the survey. 
Compared with visual impairment and blindness there is less evidence on the prevalence and causes of other types of disability. The World Health Survey [2002-2004] estimates that the prevalence of disability among those aged 18 years and above is $15.6 \%$ [7]. The prevalence of disability is higher among women, those living in rural areas and the poorest [7].

\section{Rationale for the survey}

Effective public health programmes, including for visual impairment and disability, require up-to-date evidence for priority setting, and to support specific policies and plans. Indeed, one of the objectives of the WHO Global Action Plan for Universal Eye Health is that population based data be obtained on the prevalence and causes of visual impairment [8].

There is a paucity of evidence on blindness, visual impairment and ocular morbidity at the national level in Sri Lanka. The only population-based estimates was conducted in the Kandy area of the country, reported the prevalence of blindness and visual impairment, based on best corrected visual acuity (BCVA), to be $1.1 \%$ and $5.9 \%$ respectively among those aged $\geq 40$ years [9].

Sri Lanka is a signatory to VISION2020 (a global initiative to eliminate the major causes of avoidable blindness by the year 2020) and has developed a national plan in the year 2007 for achieving the goal of elimination of avoidable blindness by 2020 . For effective monitoring and evaluation of the inputs for VISION2020, it is imperative that data be available to identify specific challenges that need to be addressed.

\section{Purpose of the survey}

To collect national data to facilitate the best use of existing resources (human, financial, infrastructure and equipment) to target the major avoidable causes of blindness in order that the goals of VISION 2020 can be achieved in Sri Lanka and to assist in planning for blindness control activities.

\section{Overall aim}

To determine the prevalence, distribution, magnitude and causes of blindness and visual impairment amongst adults aged $\geq 40$ years. This age group was selected as the prevalence of visual impairment increases exponentially with age, with $65 \%$ of all visual impairment being among those aged $\geq 50$ years [6]. Many other national surveys have also used this age group, making the data comparable.

\section{Objectives}

1. Estimate the prevalence, magnitude and causes of visual impairment and blindness

2. Determine risk factors for blindness, visual impairment, and functional low vision
3. Determine the prevalence and types of ocular morbidity in the study sample, and to investigate health-seeking behaviour among those aged $\geq 40$ years, including expenditures on health

4. Determine prevalence and type of refractive errors, spectacle coverage and barriers to accessing services

5. Assess cataract surgical services and risk factors for not accessing services

6. Assess the impact of blindness and visual impairment on quality of life and visual functioning

An additional objective was to estimate the prevalence and causes of disabilities (other than visual impairment) among adults.

\section{Methods}

Fieldwork took place over a one-year period from 2013 to 2014. A flowchart indicates the processes adopted in the survey (Figure 2).

A national steering committee under the Chairmanship of the Director General of Health Services, Ministry of Health, was set up to guide the finalization of the methodology and monitor progress of implementation.
$1 \mathrm{~A}$

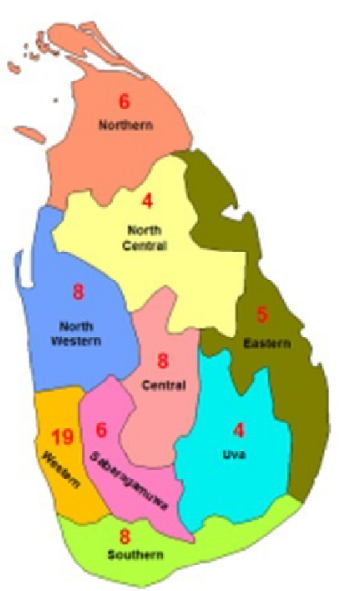

$1 \mathrm{~B}$

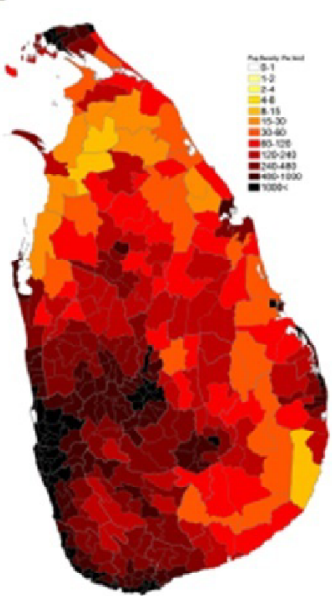

Figure 2. A Map of Sri Lanka showing location of survey clusters in each Province.

\section{Sampling}

The survey recruited a nationally representative sample of adults aged $\geq 40$ years to estimate the prevalence of blindness; and a population of adults aged $\geq 18$ years to estimate the prevalence of disability. All the nine provinces and 25 districts were included. The primary sampling units were the districts. 


\section{Sample size}

The following parameters were used to determine the sample size: prevalence of blindness (presenting vision) among those aged $\geq 40$ years based on previous data from the South Asia region - 2.5\%; confidence interval - 95\%; allowable error - 0.02 ; precision - $80 \%$; design effect - 1.5 and a response rate of $85 \%$.

The estimated sample size was 6,600. It was expected that this would provide accurate estimates for the national magnitude of blindness and visual impairment, including the major causes of blindness, ocular morbidity and disability among those aged $\geq 40$ years but was not large enough to provide accurate estimates at district level.

Table 1. Distribution of clusters included in the survey

\begin{tabular}{|c|c|c|c|}
\hline \multirow[t]{2}{*}{ Province/District } & \multicolumn{3}{|c|}{ Number of clusters } \\
\hline & Total & Rural & Urban \\
\hline Western Province & 19 & 12 & 7 \\
\hline Colombo & 8 & 2 & 6 \\
\hline Gampaha & 7 & 6 & 1 \\
\hline Kalutara & 4 & 4 & 0 \\
\hline Central Province & 8 & 7 & 1 \\
\hline Kandy & 4 & 3 & 1 \\
\hline Matale & 2 & 2 & 0 \\
\hline NuwaraEliya & 2 & 2 & 0 \\
\hline Southern Province & 8 & 8 & 0 \\
\hline Galle & 3 & 3 & 0 \\
\hline Matara & 3 & 3 & 0 \\
\hline Hambantota & 2 & 2 & 0 \\
\hline Northern Province & 6 & 6 & 0 \\
\hline Jaffna & 2 & 2 & 0 \\
\hline Kilinochchi & 1 & 1 & 0 \\
\hline Mannar & 1 & 1 & 0 \\
\hline Vavuniya & 1 & 1 & 0 \\
\hline Mullaitivu & 1 & 1 & 0 \\
\hline Eastern Province & 5 & 5 & 0 \\
\hline Batticaloa & 2 & 2 & 0 \\
\hline Ampara & 2 & 2 & 0 \\
\hline Trincomalee & 1 & 1 & 0 \\
\hline North Western Province & 8 & 8 & 0 \\
\hline Kurunegala & 5 & 5 & 0 \\
\hline Puttalama & 3 & 3 & 0 \\
\hline North Central Province & 4 & 4 & 0 \\
\hline Anuradhapura & 3 & 3 & 0 \\
\hline Polonnaruwa & 1 & 1 & 0 \\
\hline Uva Province & 4 & 4 & 0 \\
\hline Badulla & 3 & 3 & 0 \\
\hline Monaragala & 1 & 1 & 0 \\
\hline Sabaragamuwa Province & 6 & 6 & 0 \\
\hline Ratnapura & 3 & 3 & 0 \\
\hline Kegalle & 3 & 3 & 0 \\
\hline Total & 68 & 60 & 8 \\
\hline
\end{tabular}

\section{Sampling Design}

Multi-stage stratified (by province) cluster random sampling with population proportionate to size (PPS) procedures was used to identify a representative sample. Provinces were first identified and random clusters were then selected from each province. The number of clusters in each district was dependent on the size of the population in each district. Random walk was used to select participants in the selected clusters. Rural and urban areas were defined using definitions adopted by the National Census in Sri Lanka [10]. In Sri Lanka, rural regions are defined as all areas which are not administered by municipal and urban councils.

A hundred participants aged $\geq 40$ years were enumerated in each cluster. In small rural clusters where there were fewer than 100 adults available, enumeration continued in the nearest village until 100 participants were recruited. The sample was spread across 68 clusters in the country (Figure 1; Table 1), with 60 clusters being rural and the remaining urban

\section{Definitions used}

Socio-economic status:

A questionnaire was administered to collect information on the possession of different movable assets (television, computer, radio, cycle, motor cycle, car, refrigerator, air conditioner etc.). A scoring system was devised based on the presumed monetary value of each asset. Scores assigned to each asset were then cumulated to compute a family asset score. Family asset scores were categorized into four groups by calculating quartiles, yielding the upper socio-economic status (SES) as the top quartile of the family asset distribution; upper middle SES as the $2^{\text {nd }}$ quartile; lower middle SES as the $3^{\text {rd }}$ quartile and lower SES as the lowest quartile.

\section{Visual impairment and blindness:}

Blindness and moderate/severe visual impairment were defined according to WHO categories while other vision categories defined were modified from the WHO definitions [11].

Blindness: presenting visual acuity (PVA) $<20 / 400(<3 /$ 60 ) in the better eye (with glasses for distance if normally worn or unaided if glasses for distance not worn).

Severe visual impairment (SVI): presenting VA <20/200$20 / 400(<6 / 60-3 / 60)$ in the better eye.

Moderate visual impairment (Mod VI): presenting VA <20/ $63-20 / 200(<6 / 18-6 / 60)$ in the better eye.

Mild visual impairment (Mild VI): presenting VA $<20 / 40$ to $20 / 63(<6 / 12-6 / 18)$ in the better eye.

Normal/Near normal: presenting vision $\geq 20 / 40(\geq 6 / 12)$ in the better eye. 


\section{Cataract Surgical Coverage (CSC):}

This measure indicates the extent to which people who have cataract visual impairment/ blindness have accessed services. Cataract surgical coverage can be calculated at the person and eye level [12]. Calculation of $\mathrm{CSC}$ at the person level was performed for three visual acuity cut-offs: $<3 / 60,<6 / 60$ and $<6 / 18$ using the formula: $(\mathrm{x}+\mathrm{y}) /(\mathrm{x}+\mathrm{y}+\mathrm{z}) * 100$ where:

$\mathrm{x}=$ persons with unilateral pseudo/aphakia and visual impairment in contralateral eye

$\mathrm{y}=$ persons with bilateral pseudo/aphakia, regardless of acuity.

$\mathrm{z}=$ persons with $<3 / 60,<6 / 60$ and $<6 / 18$ in whom the principle cause was cataract (unilateral or bilateral)

\section{Ethical approval}

The study protocol was approved by Institutional Ethics Committees of the London School of Hygiene and Tropical Medicine (LSHTM), London, UK, the Faculty of Medicine, University of Colombo, Sri Lanka and the Indian Institute of Public Health, Hyderabad, India.

Written informed consent was obtained from all participants.

\section{Training and pilot study}

Two study teams were recruited. Both teams worked concurrently. Each team composed of one ophthalmologist, two optometrists, one team coordinator, four interviewers, one field supervisor and two drivers. The team underwent specialised training for one week at the start of the survey, led by technical experts from the International Centre for Eye Health (ICEH), LSHTM, UK. A detailed survey protocol was given to each team member. Training also covered the algorithms recommended by WHO for the diagnosis and to identify the principal cause of visual impairment. Training was followed by a pilot in two clusters near Colombo before the main study started. Feedback from the pilot studies was discussed with team members during training.

Inter-observer agreements on measurement of visual acuity and ascertaining cause of blindness/ visual impairment were undertaken for the ophthalmologists and the optometrists. This was repeated twice. Wherever poor agreement was observed (kappa <0.6), re-training was conducted. If the agreement did not improve, the poorly performing personnel were replaced with new project staff. Enumeration teams also underwent rigorous training. They were trained in the importance of an accurate denominator, how to approach the community leadership and eligible participants, administration of the disability questionnaire schedule and informed consent forms, follow up for clinical examination and recording anthropometric measurements including height and weight.

\section{Enumeration}

The project officers visited the cluster in advance and primed the local leadership on the survey. A notional map of the cluster was first prepared by the enumerators. If the cluster was big, it was first segmented into equal population sized parts and chits were drawn by a local resident to identify the segment to be included. The enumerators identified the geographical centre of the cluster after going around the circumference of the cluster with local volunteers to identify the approximate centre, and then randomly selected a direction in which to systematically enumerate households. The enumeration team serially assigned a number to each household along the selected direction and registered the names, ages and sex of all habitual occupants (residing in the cluster for more than 3 months) until 100 eligible people $\geq 40$ years were listed for each given cluster. Age was ascertained by questioning, comparison with identity cards and by using an events calendar to match the age. Three visits over two days were made to enumerated households where residents were not present before they were designated as not available. Enumeration was done on the first day while examination was done on the 1 st and $2^{\text {nd }}$ day of the visit to the cluster.

\section{Disability assessment}

The Washington Group (WG) short questionnaire was used to identify persons with self-reported disability. All household members aged $\geq 18$ years were administered the questionnaire.

Disability was defined using the WG criteria [13]. The short set of questions asks respondents to state whether they have difficulty in seeing, hearing, walking/ climbing steps, remembering/ concentrating, washing/ dressing or communicating, with answers given on a four point-scale: "no difficulty", "some difficulty", "a lot of difficulty" and "cannot do at all". Disability was defined as reporting a lot of difficulty or inability to perform the specific activity in one or more of the six domains. Functional difficulty was defined as reporting "some difficulty" in one or more of the six domains. Individuals who screened positive for functional difficulty or disability were then administered an additional questionnaire to elicit responses on activity limitations, social participation and their health concerns.

\section{Basic eye examination}

Personal and demographic data, including age, marital status, occupation, ethnicity and literacy were recorded prior to the eye examination by a trained interviewer. Participants had their blood pressure (three readings) measured (Omron R2 wrist blood pressure instrument), height and weight measured and had their distance PVA measurement with an ETDRS logarithm of minimum angle of acuity ( $\log M A R)$ tumbling "E" chart. PVA was measured in each eye separately at four metres in the open in a 
shaded area, and at one meter if necessary, in a sitting position. Any eye that could not see any letter at one meter was assessed by the ophthalmologist for finger counting, hand movements or light perception (PL/NPL) in a darkened room. If an individual could not read four out of the five optotypes of a line, it was recorded as inability to read the line. Participants who did not have their VA measured because they could not understand the test or had communication difficulties were documented as VA 'not recorded'. An ophthalmologist then conducted a basic undilated eye examination and an optometrist performed automated refraction and biometry. Participants with VA $<6 / 18$ had their BCVA measured with autorefraction results placed in a lens trial frame. Subjective refraction was done and a prescription provided where needed. They underwent detailed examination including slit lamp examination and fundus examination after dilating the pupils.

\section{Detailed eye examination}

The following participants underwent detailed slitlamp eye examination by an ophthalmologist, with 90D examination of the posterior segment after dilating the pupils: all with a PVA of $<6 / 12$ in the better eye; all who had undergone cataract surgery in one or both eyes regardless of the acuity, and all in whom an abnormality like corneal opacity, shallow anterior chamber, high IOP readings, presence of a lens opacity etc. had been noted in the basic eye examination.

Digital retinal images (Forus 3Nethra camera, Forus Health, India) were captured for those with a PVA $<6 / 12$ in the better eye when the media were clear enough.

\section{Assigning causes of visual impairment}

Causes of visual loss were assigned to all eyes with a PVA of $<6 / 12$, following the WHO algorithm designed for use in population based surveys [14]. Firstly, all the disorders contributing to visual loss in each eye, and all the relevant underlying causes were noted. Second, one main cause was selected for each eye, if more than one cause was assigned to an eye, the following were preferentially selected, if applicable: primary causes (e.g., cataract secondary to uveitis, uveitis was selected), or the disorder contributing most to vision loss. If two conditions were judged to contribute equally to visual loss, the most readily treatable was selected. If none of the conditions were treatable, the most preventable was selected. Having established a main cause for each eye, a principal cause for the person was determined, by selecting the cause in the right or left eye, following a similar logic to that described above if the causes differed between the eyes.

\section{Other procedures}

Visual functioning (VF), Quality of life and barriers to access questionnaires were administered to the following survey participants:
- Those with a PVA $<6 / 60$ in the better eye where cataract/ uncorrected refractive error was the cause of visual loss,

- Those with PVA $<6 / 18-6 / 60$ in the better eye.

- All who had undergone cataract surgery in one or both eyes irrespective of the visual outcome

- 1 in 10 individuals with normal vision. (PVA $>6 / 12$ in the better eye)

In addition all participants were administered a questionnaire asking whether they had any eye concerns in the month preceding the survey; those who responded positively were asked about their condition and their health-seeking behaviour.

\section{Service delivery}

All participants with visual impairment were referred to the nearest eye facility, where free cataract surgery had been organized for survey participants, while those with minor problems were treated at the examination site itself and reading glasses were provided free-of-cost for those in need. Persons with disability were advised to contact the nearest office of the Department of Social Services.

\section{Data management}

A record sheet was completed for each participant, which was cross-checked for errors by the ophthalmologists in the field and the project coordinator in the office. Data were subsequently entered into a customized database (with built in range and consistency checks) by an experienced data officer and independently crosschecked by a second data officer. Data cleaning and analysis were done using STATA 13.0 (StataCorp LP, Texas, USA) by a statistician at the Indian Institute of Public Health, Hyderabad.

Descriptive analyses and cross tabulations with calculation of Pearson chi squared tests were performed. Further analyses were undertaken to explore risk factors using logistic regression with generalised estimating equations to adjust for dependency in the data due to clustered sampling. All tests are two sided, and the odds ratios (OR) and 95\% confidence intervals (CI) quoted are derived from logistic regression models. To account for differential non-response, the blindness prevalence estimate was adjusted by age and sex.

The protocol and methodology used for the survey were similar to the blindness surveys conducted in Bangladesh [15], Pakistan [16] and Nigeria [17] allowing comparison.

\section{Monitoring data collection}

A team of consultant ophthalmologists from the Sri Lankan College of Ophthalmologists, the Sri Lankan 
Vision2020 Coordination Committee and representatives of Sightsavers International conducted periodic monitoring visits to the field sites and helped in maintaining quality.

\section{Acknowledgements}

We acknowledge the support from the members of the Steering Committee, the Sri Lanka College of Ophthalmologists, the Vision2020 Secretariat - Ministry of Health and Family Welfare, Sri Lanka, Sightsavers Country Office, Sri Lanka and all the supervisors and field investigators (Madhuni Wijepala, Subhashini Deshappriya, Rasika Damayanthi, Dinusha Sandamali, Ashanti de Silva, Chaturika Madushani, Menaka Rathnayake, Madusha Priyadarshini, Nadeesha Dilhani, Gaya Shanthi) and data entry operators (Sunethra Thennakoon, Kumuduni Sriyalatha) who worked with great diligence in collecting the data from the survey participants. We thank all the survey participants for giving us the time and opportunity to interact with them to collect critical data.We would like to thank Sightsavers for financial support of the overall study and CBM for financial contributions to the disability component of the survey.

\section{Conflicts of Interest}

All authors declare that they do not have any conflicts of interest.

\section{References}

1. UNDP. Human Development Report 2015. Sri Lanka country profile. http://hdr.undp.org/en/countries/profiles/ LKA. Accessed 10th June 2016.

2. Sharma K. Human development and South East Asian countries: special emphasis on India. $J$ Education Health Promotion 2013; 2: 1-4.

3. World Bank, 2008. Sri Lanka addressing the needs of an ageing population. Available at: http://siteresources. worldbank.org/IN TSR ILANKA/Resources/ LKAgingFullRep.pdf. Accessed October 212016.

4. http://www.health.gov.lk/en/index.php?option= com_content \&view $=$ article \&id=372\&Itemid $=141$. Accessed 10th June 2016.

5. Vision 2020 Sri Lanka - Structure and Activities [Internet]. [cited 2013 Dec 20]. Available from: http://www. vision2020.1k/main_areas.html. Accessed 10th June 2016.

6. Pascolini D, Mariotti SP. Global estimates of visual impairment:2010. Br J Ophthalmol 2012; 96: 614-8.

7. World Health Organization and World Bank. World Report on Disability 2010. WHO, Geneva 2011.

8. World Health Organization. Universal eye health: A global action plan 2014-2019. World Health Organization, Geneva 2013: 1-22. http://www.who.int/blindness/AP2014_19_ English.pdf. Accessed 29th June 2016.

9. Edussuriya K, Sennanayake S, Senaratne T, et al. The prevalence and causes of visual impairment in Central Sri Lanka: The Kandy Eye Study. Ophthalmology 2009; 116: $52-6$.

10. Department of Census and Statistics, Sri Lanka. Sri Lanka Census of Population and Housing 2011. http://www. statistics.gov.1k/PopHouSat/CPH2011/index.php. Accessed 20th June 2016.

11. World Health Organization. Change in the definition of blindness.http://www.who.int/blindness/Change\% 20the\%20 Definition\%20of\%20Blindness.pdf. Accessed 10th June 2016.

12. Limburg H, Foster A. Cataract surgical coverage: An indicator to measure the impact of cataract intervention programmes. Community Eye Health J 1998; 11: 3-6.

13. Madans JH, Loeb ME, Altman BA. Measuring disability and monitoring the UN Convenetion on the Rights of Persons with Disabilities: the work of the Washington Group on Disability Statistics. BMC Public Health 2011; 11(Suppl 4): S4.

14. World Health Organization. Coding instructions for the WHO/PBL Eye Examination Record (version III). PBL/ 88.1, 1988. WHO, Geneva.

15. Bourne RR, Dineen B, Modasser Ali S, Mohammed NHD, Johnson GJ. The National Blindness and Low Vision Prevalence Survey of Bangladesh: research design, eye examination methodology, and results of the pilot study. Ophthalmic Epidemiol 2002; 9: 119-32.

16. Bourne RR, Dineen B, Jadoon Z, et al. The Pakistan national blindness and visual impairment survey - research design, eye examination methodology, and results of the pilot study. Ophthalmic Epidemiol 2005; 12: 321-33.

17. Dineen B, Gilbert CE, Rabiu M, et al. The Nigerian national blindness and visual impairment survey: Rationale, objectives and detailed methodology. BMC Ophthalmol 2011; 8: 17 . 